INPLASY

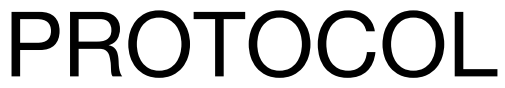

To cite: Zhao et al. The Effectiveness of Radioiodine Remnant Ablation for Low and Intermediate Risk Papillary Thyroid Microcarcinoma:A Meta-analysis. Inplasy protocol 202220030. doi:

10.37766/inplasy2022.2.0030

Received: 11 February 2022

Published: 11 February 2022

Corresponding author:

Bin Zhang

zbnuclmd@126.com

Author Affiliation:

The First Affiliated Hospital of Soochow University.

Support: Not reported.

Review Stage at time of this submission: Formal screening of search results against eligibility criteria.

Conflicts of interest:

None declared.

\section{The Effectiveness of Radioiodine Remnant Ablation for Low and Intermediate Risk Papillary Thyroid Microcarcinoma: A Meta-analysis}

Zhao, M1; Zhang, J2; Deng, S3; Zhou, Y4; Wen, R5; Lu, Y6; Zhang, $\mathrm{B}^{7}$.

Review question / Objective: The aim of this study was to evaluate whether RAl treatment can reduce the recurrence rate in LR and IR PTMC by means of a meta-analysis.

Eligibility criteria: The criteria for inclusion in the study were as follows: (1)LR and IR MPTC patients who are in a diseasefree state after surgical treatment; (2)RRA-treated vs nonRRA-treated groups; (3)reporting of tumor recurrence outcomes. Studies were excluded if (1) studies that lacked any necessary data; (2) studies that lacked a control or nonexposed group; (3) for patients with a follow-up period of less than 6 months.

INPLASY registration number: This protocol was registered with the International Platform of Registered Systematic Review and Meta-Analysis Protocols (INPLASY) on 11 February 2022 and was last updated on 11 February 2022 (registration number INPLASY202220030).

\section{INTRODUCTION}

Review question / Objective: The aim of this study was to evaluate whether RAl treatment can reduce the recurrence rate in LR and IR PTMC by means of a metaanalysis.
Condition being studied: The Effectiveness of Radioiodine Remnant Ablation for Low and Intermediate Risk Papillary Thyroid Microcarcinoma. 


\section{METHODS}

Search strategy: We used the following search method to search the PubMed, Embase, and Cochrane library : ("remnant ablation"[tiab] OR "radioiodine"[tiab] OR "radioactive iodine"[tiab] OR "iodine 131" [tiab] OR "iodine-131"[tiab] OR "RAl" [tiab]) AND ("papillary thyroid microcarcinoma" [tiab] OR "papillary microcarcinoma" [tiab] OR "thyroid microcarcinoma" [tiab] OR "PTMC"[tiab]).

Participant or population: Low-risk and intermediate-risk papillary thyroid microcarcinoma patients.

Intervention: Radioactive iodine (RAI) remnantablation.

\section{Comparator: Not reported.}

Study designs to be included: The PubMed, Embase, Cochrane Library and Web of Science were searched for studies that compared the effectiveness after surgery with or without RAl remnant ablation treatment

Eligibility criteria: The criteria for inclusion in the study were as follows: (1)LR and IR MPTC patients who are in a disease-free state after surgical treatment; (2)RRAtreated vs non-RRA-treated groups; (3)reporting of tumor recurrence outcomes. Studies were excluded if (1) studies that lacked any necessary data; (2) studies that lacked a control or non-exposed group; (3) for patients with a follow-up period of less than 6 months.

Information sources: The PubMed, Embase, Cochrane Library and Web of Science were searched for studies that compared the effectiveness after surgery with or without RAI remnant ablation treatment

Main outcome(s): Not reported.

Quality assessment / Risk of bias analysis: The Newcastle-Ottawa Scale (NOS) was used by two reviewers to independently evaluate the quality of retrospective (casecontrol and cohort) researches.

Strategy of data synthesis: Two of the researchers independently assessed all the selected papers to see if the articles met the requirements. The two authors resolve any differences that arise through discussion. If no agreement is reached, the other authors are contacted, and a decision by popular election is taken.

Subgroup analysis: Not reported.

Sensitivity analysis: Two sensitivity analyses were carried out to examine the stability of the pooled effects by excluding one or two individual studies to ascertain their impact on the pooled RR.

Language: No restriction.

Country(ies) involved: China.

Keywords: papillary thyroid microcarcinoma, low-risk, intermediaterisk, radioiodine remnant ablation, metaanalysis.

Contributions of each author:

Author 1 - Min Zhao.

Email: sdqzm0817@163.com

Author 2 - Jing Zhang.

Email: zjzzglsnzcx@126.com

Author 3 - Shengming Deng.

Email: dshming@163.com

Author 4 - Yeye Zhou.

Email: zhouyeye23@163.com

Author 5 - Runze Wen.

Email: wenrunze2021@163.com

Author 6 - Yixing Lu.

Email: 634722296@qq.com

Author 7 - Bin Zhang.

Email: zbnuclmd@126.com

$M Z$ designed the research study. $M Z, J Z$, $R Z W$ and $Y X L$ collected data and performed data analysis. $M Z$ drafted the manuscript and designed the figures. $M Z$, JZ, RZW and YXL contributed to the editing of the final manuscript. SMD, YYZ and BZ reviewed the manuscript. 